\title{
Resolution of a family of Galois embedding problems with cyclic kernel
}

Montserrat Vela

\begin{abstract}
In this paper we compute the obstruction and the solutions of cyclic embedding problems given by

$$
(E): \quad 0 \rightarrow \mathbb{Z} / n \mathbb{Z} \rightarrow E \rightarrow \Gamma=\mathbb{Z} / n \mathbb{Z} \times \cdots \times(\cdots) / n \mathbb{Z} \rightarrow 0,
$$

with $\mathbb{Z} / n \mathbb{Z}$ trivial $\Gamma$-modulo, finding adequate representations of $\Gamma$ in the automorphisms group of a generalized Clifford algebra.
\end{abstract}

\section{Introduction}

In [7] we have studied Galois embedding problems given by central extensions with cyclic kernel. In particular, we have computed an expression for the obstruction to the solvability of these embedding problems in terms of Galois symbols, generalizing the formula given by Fröhlich in [3]. To compute this obstruction, we associate to the embedding problem a representation $t$ of $\Gamma$ in the group of graded automorphisms of an adequate generalized Clifford algebra with an admissible norm.

We have too given a method for constructing the solutions when these problems are solvable, generalizing the results obtained by Crespo in [1] and [2]. We obtain a way to compute a solution of the solvable embedding problems of the form $L(\sqrt[n]{\gamma})$ where $\gamma$ is a coordinate of the norm of an adequate element in a generalized Clifford algebra.

Using this study we solve now a complete family of problems.

We recall in short the notations and results used in [7] that we need to do this study.

2000 Mathematics Subject Classification: 12F12, 11R32, 11E88.

Keywords: Galois embedding problems, generalized Clifford algebras. 
Let $n \geq 2$ be an integer, $K$ be a field of characteristic not dividing $n$ containing the group $\mu_{n}$ of $n$th roots of unity, and fix $\omega$ a primitive $n$th root of unity. We denote by $l g$ the group homomorphism $l g: \mu_{n} \rightarrow \mathbb{Z} / n \mathbb{Z}$ where $\lg \left(\omega^{i}\right)=i$, the separable closure of $K$ is denoted by $K^{s e p}$ and the absolute Galois group by $G_{K}=\operatorname{Gal}\left(K^{\text {sep }} / K\right)$.

We consider cyclic embedding problems given by

$$
\begin{gathered}
L=K\left(\sqrt[n]{a_{1}}, \ldots, \sqrt[n]{a_{m}}\right) / K, \quad \Gamma \simeq \mathbb{Z} / n \mathbb{Z} \times \cdots \times \times \mathbb{Z} / n \mathbb{Z}, \\
(E): \quad 0 \rightarrow \mathbb{Z} / n \mathbb{Z} \rightarrow E \rightarrow \Gamma \rightarrow 0,
\end{gathered}
$$

with $\mathbb{Z} / n \mathbb{Z}$ trivial $\Gamma$-module. Let $j: G_{K} \rightarrow \Gamma$ be the surjective homomorphism corresponding to $L / K$. We consider the homomorphism between the cohomology groups $j_{2}^{*}: H^{2}(\Gamma, \mathbb{Z} / n \mathbb{Z}) \rightarrow H^{2}\left(G_{K}, \mathbb{Z} / n \mathbb{Z}\right)$ induced by $j$ and let $\varepsilon$ be the element in $H^{2}(\Gamma, \mathbb{Z} / n \mathbb{Z})$ corresponding to $(E)$.

The embedding problem given by $L / K, \Gamma,(E)$ is solvable if and only if $j_{2}^{*}(\varepsilon)=0$. The element $j_{2}^{*}(\varepsilon)$ is called the obstruction to the solvability of the embedding problem.

We use the term generalized Clifford algebra to refer to a finite dimensional $K$-algebra generated by elements $\left\{e_{1}, \ldots, e_{m}\right\}$ with relations $e_{i}^{n}=$ $a_{i} \in K^{*}$ and $e_{i} e_{j}=\omega e_{j} e_{i}$ if $i<j$. We denote this algebra as $C(V)$ for $V=<e_{1}, \ldots, e_{m}>_{K}$. We can make $C(V)$ into a $\mathbb{Z} / n \mathbb{Z}$-graded $K$-algebra

$$
C(V)=C(V)_{0} \oplus \cdots \oplus C(V)_{n-1}
$$

by setting

$$
C(V)_{l}=<e_{1}^{\varepsilon_{1}} \cdots e_{m}^{\varepsilon_{m}} \mid \varepsilon_{1}+\cdots+\varepsilon_{m} \equiv l \quad(\bmod n)>_{K} .
$$

If $m$ is odd the invariant of $C(V)$ is

$$
a=(-1)^{\frac{(n-1)(m-1)}{2}} a_{1} a_{2}^{-1} \cdots a_{m-1}^{-1} a_{m}
$$

and if $m$ is even the invariant is

$$
a=(-1)^{\frac{(n-1) m}{2}} a_{1}^{-1} a_{2} \cdots a_{m-1}^{-1} a_{m} .
$$

The Brauer invariant of $C(V)$ defined in [7, Definition 4.2] is the class in the Brauer group of $C(V)$ if $m$ is even or of $C(V)_{0}$ if $m$ is odd.

We call the norm of the generalized Clifford algebra $C(V)$ the map $N$ : $C(V) \longrightarrow C(V)$ given by $N(z):=\beta(z) z$ where

$\beta\left(\sum \alpha_{\varepsilon_{1}, \cdots, \varepsilon_{m}} e_{1}^{\varepsilon_{1}} \cdots e_{m}^{\varepsilon_{m}}\right)=\sum \alpha_{\varepsilon_{1}, \cdots, \varepsilon_{m}}^{n-1} e_{m}^{\varepsilon_{m}(n-1)} \cdots e_{1}^{\varepsilon_{1}(n-1)}, \quad \alpha_{\varepsilon_{1}, \cdots, \varepsilon_{m}} \in K$. 
The norm $N$ restricted to the subgroup

$$
\begin{aligned}
F(C(V)):=\left\{x \in C(V)^{*}\right. & \text { homog. s.t. } N(x) \in K^{*} \\
& \text { and } \beta(x y)=\beta(y) \beta(x) \forall y \in C(V)\}
\end{aligned}
$$

is multiplicative.

Let $A$ be a subgroup of $F(C(V))$ such that $K^{*} \subset A$. An admissible norm in $A$ is a map $\mathcal{N}: A \longrightarrow K^{*}$ such that for $a, a_{1}, a_{2} \in A$,

$$
\mathcal{N}(a) \in K^{*}, \quad \mathcal{N}\left(a_{1} a_{2}\right)=\mathcal{N}\left(a_{1}\right) \mathcal{N}\left(a_{2}\right) \quad \text { and } \quad \mathcal{N}(\lambda)=\lambda^{n} \text { if } \lambda \in K .
$$

In particular, for $A=F(C(V))$, the norm $N$ is an admissible norm.

We recall that, given a profinite group $\Gamma$, a representation of $\Gamma$ over $K$ is the pair given by

(1) A generalized Clifford algebra $C_{t}=C\left(V_{t}\right)$ over $K$ with an admissible norm $(\mathcal{N}, A)$ together with

(2) A continuous homomorphism $t: \Gamma \rightarrow O\left(C_{t}\right) \subset \operatorname{Autgr}\left(C_{t}\right)$, where $O\left(C_{t}\right)=$ $\varphi(A)$ with the homomorphism $\left\{\right.$ Homogeneous elements of $\left.C(V)^{*}\right\} \stackrel{\varphi}{\rightarrow}$ $\operatorname{Autgr}(C(V))$ where, for all $x \in C(V)$ homogeneous,

(a) $\varphi(s)(x)=s x s^{-1} \quad$ if $\operatorname{dim}(V)$ is even,

(b) $\varphi(s)(x)=\omega^{\partial(s) \partial(x)} s x s^{-1}$ if $\operatorname{dim}(V)$ is odd (where $\partial$ denotes the degree).

Given a representation $t: \Gamma \rightarrow O\left(C_{t}\right)$, we use the term twisted algebra of $C_{t}$ by $t$ to refer to the $K$-algebra $\mathfrak{C}_{t}$ corresponding to the element

$$
\begin{aligned}
\alpha: \Gamma & \stackrel{t}{\rightarrow} O\left(C_{t}\right) \rightarrow O\left(C_{t} \otimes L\right) \subset \operatorname{Autgr}\left(C_{t} \otimes L\right), \\
\sigma & \mapsto t(\sigma)_{L}(x \otimes \lambda)=t(\sigma)(x) \otimes \lambda
\end{aligned}
$$

of $H^{1}\left(\Gamma, \operatorname{Aut}\left(C_{t} \otimes L\right)\right)$ by the bijection given in [3, III.2] (see too [6, Chap. X]). We have $\mathfrak{C}_{t} \simeq\left(C_{t} \otimes L\right)^{\Gamma}$ where $\Gamma$ acts on $C_{t} \otimes L$ via $t \otimes g a l$, i.e., $\sigma(x \otimes \lambda)=$ $t(\sigma)(x) \otimes \sigma(\lambda)$ and the isomorphism $g: \mathfrak{C}_{t} \otimes L \stackrel{\sim}{\rightarrow} C_{t} \otimes L$, where $\Gamma$ acts on $C_{t} \otimes L$ via $1 \otimes g a l$, that is, $\sigma(x \otimes \lambda)=x \otimes \sigma(\lambda)$. This morphism satisfies $g^{-1} g^{\sigma}=t(\sigma)$ for each $\sigma \in \Gamma$.

We have obtained, from the diagram of [7, Proposition 5.3] the exact sequence

$$
1 \rightarrow \mathbb{Z} / n \mathbb{Z} \rightarrow \operatorname{Ker}_{K^{s e p}} \rightarrow O\left(C_{t} \otimes_{K} K^{s e p}\right) \rightarrow 1
$$

where $\mathcal{N}_{K^{\text {sep }}}$ denotes the extension of $\mathcal{N}$ to the subgroup $A_{K^{\text {sep }}}$ of

$$
F\left(C(V) \otimes_{K} K^{s e p}\right)
$$

generated by $A$. 
Composing the representation $t$ with

$$
O\left(C_{t}\right) \rightarrow O\left(C_{t} \otimes_{K} K^{s e p}\right) \subset \operatorname{Autgr}\left(C_{t} \otimes_{K} K^{s e p}\right)
$$

we obtain

$$
\begin{aligned}
& \Gamma \\
& \downarrow t \\
& O\left(C_{t}\right) \\
& 1 \rightarrow \mathbb{Z} / n \mathbb{Z} \rightarrow \operatorname{Ker}_{K^{\text {sep }}} \rightarrow O\left(C_{t} \otimes_{K} K^{\text {sep }}\right) \rightarrow 1
\end{aligned}
$$

that is an extension of $\Gamma$ by $\mathbb{Z} / n \mathbb{Z}$ and so, an element of $H^{2}(\Gamma, \mathbb{Z} / n \mathbb{Z})$. The analogue to the second Stiefel-Whitney class, denoted by $s_{t}$, is this element of $H^{2}(\Gamma, \mathbb{Z} / n \mathbb{Z})$. If $\Gamma=G_{K}$, the element $s_{t}$ belongs to

$$
H^{2}\left(G_{K}, \mathbb{Z} / n \mathbb{Z}\right)=\operatorname{Br}_{n}(K) .
$$

To compute the obstruction to the embedding problem, we need to find a representation $t$ of $\Gamma$ so that $\varepsilon=s_{t}$. From such a representation we have the Formula [7, 6.7]:

Formula 1.1. The obstruction $j_{2}^{*}$ is

$$
\begin{array}{ll}
\text { (a) } j_{2}^{*}\left(s_{t}\right)=\left[\mathfrak{C}_{t}\right]-\left[C_{t}\right]+P \mathcal{N}_{2}[t \circ j] & \text { if } \operatorname{dim}\left(V_{t}\right) \text { is even, } \\
\text { (b) } j_{2}^{*}\left(s_{t}\right)=\left[\mathfrak{C}_{t}\right]-\left[C_{t}\right]-\left(a_{t}, b_{t \circ j}\right)+P \mathcal{N}_{2}[t \circ j] & \text { if } \operatorname{dim}\left(V_{t}\right) \text { is odd, }
\end{array}
$$

where $\mathfrak{C}_{t}$ is the twisted algebra of $C_{t}$ by $t, a_{t}$ is the invariant of $C_{t}, b_{t} \in$ $K^{*} / K^{*^{n}}$ is the element corresponding to $d(t) \in \operatorname{Hom}(\Gamma, \mathbb{Z} / n \mathbb{Z})$ defined by $\sigma \mapsto d(t)(\sigma)=\partial\left(s(\sigma)\right.$ ) (where $s(\sigma) \in \varphi^{-1}(t(\sigma))$ ) by Kummer theory and $\operatorname{PN}_{2}[t] \in B r_{n}(K)$ is the class of the cocycle

$$
(\sigma, \tau) \mapsto \lg \frac{\sigma(\sqrt[n]{\mathcal{N}(s(\tau))})}{\sqrt[n]{\mathcal{N}(s(\tau))}}
$$

and can be expressed as a sum of Galois symbols ([7, Proposition 5.6]).

If we know that the embedding problem is solvable, according [7, Theorem 8.2], the steps to be followed in order to obtain an element $\gamma$ such that $L(\sqrt[n]{\gamma})$ is a solution are:

1. Find a "good" representation of degree 0 such that $s_{t}=\varepsilon$.

2. Write down explicitly the isomorphism $g: C_{L} \rightarrow \mathfrak{C}_{L}$ over $L$ such that $g^{-1} g^{\sigma}=t(\sigma) \forall \sigma \in \Gamma$. 
3. Determine an isomorphism $f: C \rightarrow \mathfrak{C}$ over $K$ such that the element $z$ defined in [7, Theorem 8.2] be different from 0.

4. Compute the expression of the element $z$ in the basis of $\mathfrak{C}_{L}$.

5. Compute the norm $N(z) \in \mathfrak{C}_{L}$ and consider a non-zero coordinate $\alpha$ of it.

6. Find $\eta \in L$ such that $\eta^{-\sigma} \eta=n_{\sigma}^{-1}, \forall \sigma \in \Gamma$.

7. Compute the element $\gamma=\eta \alpha^{-1}$.

(In fact, here we compute $\gamma^{-1}$ because of the difficulty to compute $\alpha^{-1}$ from $\alpha$ and the fact that the field $K\left(\sqrt[n]{\gamma^{-1}}\right)$ is equal to $K(\sqrt[n]{\gamma})$.

In this way we obtain all solutions of the embedding problem because if $L(\sqrt[n]{\gamma})$ is a solution of the solvable embedding problem $L / K, \Gamma, E$, the set $\{\sqrt[n]{r \gamma} \mid r \in K\}$ contains all proper and improper solutions.

\section{Representations for a family of problems}

Let $n$ be an odd integer and $L=K\left(\sqrt[n]{a_{1}}, \ldots, \sqrt[n]{a_{m}}\right)$ be a Galois extension of $K$ with Galois group

$$
\Gamma=\operatorname{Gal}(L / K) \simeq \mathbb{Z} / n \mathbb{Z} \times \cdots \times+\frac{m}{\cdots} \times \mathbb{Z} \mathbb{Z} .
$$

In particular we suppose $a_{i} \notin K^{d}$ for all $d \mid n, d \neq 1$ and the $a_{i}$ are independent in $K^{*} / K^{*^{n}}$.

We compute the obstruction to the solvability of embedding problems given by

$$
L / K, \Gamma,(E): \quad 0 \rightarrow \mathbb{Z} / n \mathbb{Z} \rightarrow E \rightarrow \Gamma \rightarrow 0,
$$

with $\mathbb{Z} / n \mathbb{Z}$ trivial $\Gamma$-module, that is, where $E$ is a central extension of $\Gamma$ by $\mathbb{Z} / n \mathbb{Z}$. Equivalently, we can consider the problem given by $L / K, \Gamma, \varepsilon$, where $\varepsilon \in H^{2}(\Gamma, \mathbb{Z} / n \mathbb{Z})$ is the element corresponding to $(E)$. Let $\sigma_{i}(1 \leq i \leq m)$ be the automorphisms of $L$ given by

$$
\sigma_{i}\left(\sqrt[n]{a_{j}}\right)=\omega^{\delta_{i j}} \sqrt[n]{a_{j}}
$$

such that $\Gamma=<\sigma_{1}, \ldots, \sigma_{m}>$.

We look for a representation $t$ of $\Gamma$ over $K$ such that

$$
\varepsilon=s_{t} \in H^{2}(\Gamma, \mathbb{Z} / n \mathbb{Z})
$$

where $s_{t}$ is the analogue to the second Stiefel-Whitney class. 
We begin by defining a generalization of Galois symbols.

Definition 2.1. Let $L / K$ be a Galois extension and $\Gamma=\operatorname{Gal}(L / K)$. For $a \in L^{*^{n}} \cap K^{*}$ we denote by

$$
\chi_{a} \in \operatorname{Hom}(\Gamma, \mathbb{Z} / n \mathbb{Z})=H^{1}(\Gamma, \mathbb{Z} / n \mathbb{Z})
$$

the corresponding element by Kummer isomorphism relative to $L$, that is

$$
\chi_{a}(\sigma)=\lg \left(\frac{\sigma(\sqrt[n]{a})}{\sqrt[n]{a}}\right)
$$

For $a, b \in L^{*^{n}} \cap K^{*}$ we define

$$
(a, b)_{L}:=\chi_{a} \cup \chi_{b}
$$

where the cup-product is considered via the multiplication in $\mathbb{Z}$.

Proposition 2.2. These symbols have the next properties:

1. They are bilineal, that is,

$$
\left(a a^{\prime}, b\right)_{L}=(a, b)_{L}+\left(a^{\prime}, b\right)_{L} \text { and }\left(a, b b^{\prime}\right)_{L}=(a, b)_{L}+\left(a, b^{\prime}\right)_{L} .
$$

2. If $n$ is odd $(a, a)_{L}=0$ and $(a, b)_{L}+(b, a)_{L}=0$.

Proof. 1. As $\chi_{a a^{\prime}}(\sigma)=\chi_{a}(\sigma)+\chi_{a^{\prime}}(\sigma)$ for all $\sigma \in \Gamma$, they are bilineal.

2. For the first equality our goal is to prove that $\chi_{a} \cup \chi_{a}$ is a coboundary, that is, that there exists a map $h: \Gamma \rightarrow \mathbb{Z} / n \mathbb{Z}$ such that

$$
\chi_{a}(\sigma) \chi_{a}(\tau)=-h(\sigma \tau)+h(\sigma)+h(\tau) \quad \forall \sigma, \tau \in \Gamma .
$$

If we consider the morphism

$$
\psi_{a}: G_{K} \rightarrow \mathbb{Z} / n \mathbb{Z}, \sigma \mapsto \psi_{a}(\sigma)=\frac{\sigma(\sqrt[n]{a})}{\sqrt[n]{a}},
$$

by properties of Galois symbols ([6, XIV.2, Prop. 4] and [7, Lemma 4.1]), $\psi_{a} \cup \psi_{a}$ is a coboundary. Therefore, there exists a map $\eta: G_{K} \rightarrow \mathbb{Z} / n \mathbb{Z}$ such that

$$
\psi_{a}(\sigma) \psi_{a}(\tau)=-\eta(\sigma \tau)+\eta(\sigma)+\eta(\tau) \quad \text { for all } \sigma, \tau \in G_{K}
$$

Let $j: G_{K} \rightarrow \Gamma$ be the surjective morphism and we fix $u$ a section of $j$. We define the map $h(\sigma):=\eta(u(\sigma))$ for $\sigma \in \Gamma$. This map satisfies for $\sigma, \tau \in \Gamma$, $\psi_{a}(u(\sigma)) \psi_{a}(u(\tau))=-\eta(u(\sigma \tau))+\eta(u(\sigma))+\eta(u(\tau))=-h(\sigma \tau)+h(\sigma)+h(\tau)$ and, since $\psi_{a}(u(\sigma))=\chi_{a}(\sigma)$ for all $\sigma \in \Gamma$ because $a \in L$, we get the relation $(a, a)_{L}=0$.

From the previous results, we have

$$
0=(a b, a b)_{L}=(a, a)_{L}+(a, b)_{L}+(b, a)_{L}+(b, b)_{L}=(a, b)_{L}+(b, a)_{L}
$$


We now define some representations:

Definition 2.3. 1. For the following representations we consider the generalized Clifford algebra $C(e)$ such that $e^{n}=1$ and $\mathcal{N}=N$ (norm of the algebra):

(a) For $i \in\{1, \ldots, m\}$ we define

$$
\begin{aligned}
\phi_{i}: \Gamma & \longrightarrow \operatorname{Autgr}(C(e)) \\
\sigma_{i} & \longrightarrow \varphi(e) \\
\sigma_{r} & \longrightarrow 1 \quad \text { if } r \neq i
\end{aligned}
$$

and

$$
\begin{aligned}
\phi_{i}^{k}: \Gamma & \longrightarrow \operatorname{Autgr}(C(e)) \\
\sigma_{i} & \longrightarrow \varphi\left(e^{k}\right) \\
\sigma_{r} & \longrightarrow 1 \quad \text { if } r \neq i
\end{aligned}
$$

(b) For $i_{1}, \cdots, i_{k} \in\{1, \ldots, m\}$ all different we define

$$
\begin{aligned}
\phi_{i_{1}} \cdots \phi_{i_{k}}: \Gamma & \longrightarrow \operatorname{Autgr}(C(e)) \\
\sigma_{i_{j}} & \longrightarrow \varphi(e) \quad \text { for } j=1, \ldots k \\
\sigma_{r} & \longrightarrow 1 \quad \text { if } r \neq i_{j},
\end{aligned}
$$

and in the same way the representation

$$
\phi_{i_{1}}^{m_{i_{1}}} \cdots \phi_{i_{k}}^{m_{i_{k}}} .
$$

2. We now consider the generalized Clifford algebra $C(v)$ with $v^{n}=\omega$, and define the representation:

$$
\begin{aligned}
\psi_{i}: \Gamma & \longrightarrow \operatorname{Autgr}(C(v)) \\
\sigma_{i} & \longrightarrow \varphi(v) \\
\sigma_{r} & \longrightarrow 1 \quad \text { if } r \neq i
\end{aligned}
$$

with

$$
A_{\psi_{i}}=\left\{\lambda v^{r} \mid \lambda \in K, r \in\{0, \ldots, n-1\}\right\}
$$

and the admissible norm $\mathcal{N}(v)=\omega^{-1} N(v)=1$.

We also define the representation

$$
\begin{aligned}
\psi_{i}^{k}: \Gamma & \longrightarrow \text { Autgr }(C(v)) \\
\sigma_{i} & \longrightarrow \varphi\left(v^{k}\right) \\
\sigma_{r} & \longrightarrow 1 \quad \text { if } r \neq i
\end{aligned}
$$

As in the previous case we define the representations:

$$
\psi_{i_{1}} \cdots \psi_{i_{k}} \quad \text { and } \quad \psi_{i_{1}}^{m_{i_{1}}} \cdots \psi_{i_{k}}^{m_{i_{k}}} \text {. }
$$


Proposition 2.4. For these representations we have:

1. $s_{\phi_{i}}=0$ and $b_{\phi_{i}}=a_{i}$.

$s_{\phi_{i}^{k}}=0$ and $b_{\phi_{i}^{k}}=a_{i}^{k}$.

$s_{\phi_{i_{1}} \cdots \phi_{i_{k}}}=0$ and $b_{\phi_{i_{1}} \cdots \phi_{i_{k}}}=a_{i_{1}} \cdots a_{i_{k}}$.

$s_{\phi_{i_{1}}^{m_{i_{1}} \ldots \phi_{i_{k}}} m_{i_{k}}}=0$ and $\quad b_{\phi_{i_{1}}^{m_{i_{1}} \ldots \phi_{i_{k}}}}^{m_{i_{k}}}=a_{i_{1}}^{m_{i_{1}}} \cdots a_{i_{k}}^{m_{i_{k}}}$.

2. We denote $s_{\psi_{i}}=\left(a_{i}\right) \in H^{2}(\Gamma, \mathbb{Z} / n \mathbb{Z})$ (see Remark 2.5). Then

$$
\begin{aligned}
& b_{\psi_{i}}=a_{i} . \\
& s_{\psi_{i}^{k}}=\left(a_{i}^{k}\right) \text { and } b_{\psi_{i}^{k}}=a_{i}^{k} . \\
& s_{\psi_{i_{1}} \cdots \psi_{i_{k}}}=\left(a_{i_{1}} \cdots a_{i_{k}}\right) \text { and } b_{\psi_{i_{1}} \cdots \psi_{i_{k}}}=a_{i_{1}} \cdots a_{i_{k}} . \\
& s_{\psi_{i_{1}}^{m_{i_{1}} \cdots \psi_{i_{k}}}}^{m_{i_{k}}}=\left(a_{i_{1}}^{m_{i_{1}}} \cdots a_{i_{k}}^{m_{i_{k}}}\right) \text { and } b_{\psi_{i_{1}}^{m_{i_{1}} \cdots \psi_{i_{k}}}}^{m_{i_{k_{k}}}}=a_{i_{1}}^{m_{i_{1}}} \cdots a_{i_{k}}^{m_{i_{k}}} .
\end{aligned}
$$

Proof. To prove these results it is only necessary to apply the definition of analogue to Stiefel-Whitney class $s_{t}$.

Remark 2.5. The element $\left(a_{i}\right)$ defined before coincides, if $n$ is a prime number with the element $\left(\left(a_{i}\right)\right)$ defined in [5] because both are defined by the same 2-cocycle.

Proposition 2.6. Let $j$ be the surjective morphism $j: G_{K} \rightarrow \Gamma$ and $j_{2}^{*}$ the homomorphism between the cohomology groups induced by $j$. For the above representations we have:

1. $j_{2}^{*}\left(s_{\phi_{i}}\right)=0, \quad j_{2}^{*}\left(s_{\phi_{i}^{k}}\right)=0$.

$j_{2}^{*}\left(s_{\phi_{i} \phi_{k}}\right)=0, \quad j_{2}^{*}\left(s_{\phi_{i_{1}} \cdots \phi_{i_{k}}}\right)=0, \quad j_{2}^{*}\left(s_{\phi_{i_{1}} m_{i_{1}} \ldots \phi_{i_{k}}}^{m_{i_{k}}}\right)=0$.

2. $j_{2}^{*}\left(s_{\psi_{i}}\right)=j_{2}^{*}\left(\left(a_{i}\right)\right)=\left(a_{i}, \omega\right), \quad j_{2}^{*}\left(s_{\psi_{i}^{k}}\right)=j_{2}^{*}\left(\left(a_{i}^{k}\right)\right)=\left(a_{i}^{k}, \omega\right)$. $j_{2}^{*}\left(s_{\psi_{i} \psi_{k}}\right)=j_{2}^{*}\left(\left(a_{i} a_{k}\right)\right)=\left(a_{i} a_{k}, \omega\right), j_{2}^{*}\left(s_{\psi_{i_{1}} m_{i_{1}} \ldots \psi_{i_{k}}}^{m_{i_{k}}}\right)=\left(a_{i_{1}}^{m_{i_{1}}} \cdots a_{i_{k}}^{m_{i_{k}}}, \omega\right)$.

3. $j_{2}^{*}\left(s_{\phi_{i} \hat{\otimes} \phi_{j}}\right)=\left(a_{i}, a_{j}\right)$, that is $j_{2}^{*}\left(\left(a_{i}, a_{j}\right)_{L}\right)=\left(a_{i}, a_{j}\right)$.

Proof. If $t, t_{1}, t_{2}$ are representations of $\Gamma$, it is known that $s_{t \circ j}=j_{2}^{*}\left(s_{t}\right)$ and therefore $j_{2}^{*}\left(s_{t_{1} \hat{\otimes} t_{2}}\right)=j_{2}^{*}\left(s_{t_{1}}\right)+j_{2}^{*}\left(s_{t_{2}}\right)+\left(b_{t_{1}}, b_{t_{2}}\right)([7$, Proposition 5.5]).

1. For $t$ a representation of 1 ., we have by $1.1 j_{2}^{*}\left(s_{t}\right)=\left[\mathfrak{C}_{t}\right]-\left[C_{t}\right]-\left(a_{t}, b_{t}\right)$ where $\left[C_{t}\right]=[C(e)]=0$ and the invariant $a_{t}=1$. The twisted algebra $\mathfrak{C}_{t}$ is generated only by an element, so $\left[\mathfrak{C}_{t}\right]=0$ and $j_{2}^{*}\left(s_{t}\right)=0$.

2. For $t$ a representation of 2., we have, as before, $j_{2}^{*}\left(s_{t}\right)=\left[\mathfrak{C}_{t}\right]-\left[C_{t}\right]-$ $\left(a_{t}, b_{t}\right)$ where $\left[C_{t}\right]=[C(v)]=0$ and $\left[\mathfrak{C}_{t}\right]=0$. But in this case, its invariant $a_{t}=\omega$. Thus, $j_{2}^{*}\left(s_{t}\right)=\left(b_{t}, \omega\right)$.

3. It is a consequence of the above equalities. 
Theorem 2.7. A representation $t$ of $\Gamma$ such that

$$
s_{t}=\sum_{1 \leq i<j \leq m} \lambda_{i, j}\left(a_{i}, a_{j}\right)_{L}+\mu\left(a_{i_{1}}^{m_{i_{1}}} \cdots a_{i_{k}}^{m_{i_{k}}}\right), \quad \mu=0 \text { or } 1
$$

is $t=\rho \hat{\otimes} \mu\left(\rho^{\prime} \hat{\otimes} \rho^{\prime \prime}\right)$, where:

1. The representation $\rho$ satisfies

$$
s_{\rho}=\sum_{1 \leq i<j \leq m} \lambda_{i, j}\left(a_{i}, a_{j}\right)_{L}=\sum_{1 \leq i<j \leq m}\left(a_{i}, a_{j}^{\lambda_{i, j}}\right)_{L}
$$

and it is $\rho=\rho_{1} \hat{\otimes} \cdots \hat{\otimes} \rho_{m-1}$ for

$$
\begin{aligned}
\rho_{1} & =\phi_{1} \hat{\otimes} \phi_{2}^{\lambda_{1,2}} \cdots \phi_{m}^{\lambda_{1, m}}, \\
\rho_{i} & =\phi_{i} \hat{\otimes} \phi_{i+1}^{\lambda_{i, i+1}} \cdots \phi_{m}^{\lambda_{i, m}} \hat{\otimes} \phi_{i} \phi_{i+1}^{\lambda_{i, i+1}} \cdots \phi_{m}^{\lambda_{i, m}} \hat{\otimes} \phi_{1}^{d_{i, 1}} \cdots \phi_{m}^{d_{i, m}}, \quad i>1 .
\end{aligned}
$$

with the elements $d_{i, k}$ :

$$
\begin{aligned}
d_{2,1} & =1, \quad d_{i+1,1}=1+\sum_{r=2}^{i} d_{r, 1} \\
d_{2,2} & =\lambda_{1,2} \quad d_{i+1,2}=\lambda_{1,2}+2+\sum_{r=2}^{i} d_{r, 2} \quad \text { if } i>1 \\
d_{i+1, k} & =\lambda_{1, k}+2 \sum_{r=2}^{i-1} \lambda_{r, k}+2+\sum_{r=2}^{i} d_{r, k} \quad \text { if } 2<k \leq i \\
d_{i+1, k} & =\lambda_{1, k}+2 \sum_{r=2}^{i} \lambda_{r, k}+\sum_{r=2}^{i} d_{r, k} \quad \text { if } k>i
\end{aligned}
$$

where $\lambda_{k, k}=1$ and $\lambda_{i, j}=0$ if $i>j$.

2. The representation $\rho^{\prime}$ is

$$
\rho^{\prime}=\psi_{i_{1}}^{m_{i_{1}}} \cdots \psi_{i_{k}}^{m_{i_{k}}}
$$

3. The representation $\rho^{\prime \prime}$ is

$$
\rho^{\prime \prime}=\phi_{i_{1}}^{m_{i_{1}}} \cdots \phi_{i_{k}}^{m_{i_{k}}} \hat{\otimes} \phi_{1}^{d_{m, 1}} \cdots \phi_{m}^{d_{m, m}}
$$

where the elements $d_{m+1, i}$ are the ones defined above.

Remark 2.8. If $n$ is prime, Theorem 2.7 gives a representation $t$ such that $s_{t}=\varepsilon$ for all elements $\varepsilon \in H^{2}(\Gamma, \mathbb{Z} / n \mathbb{Z}$ ) (see the decomposition done by Massy [5]). 
Theorem 2.9. The obstruction to the solvability of the embedding problem given by $L / K, \Gamma$ and $s_{t} \in H^{2}(\Gamma, \mathbb{Z} / n \mathbb{Z})$ for

$$
s_{t}=\sum_{1 \leq i<j \leq m} \lambda_{i, j}\left(a_{i}, a_{j}\right)_{L}+\mu\left(a_{i_{1}}^{m_{i_{1}}} \cdots a_{i_{k}}^{m_{i_{k}}}\right), \quad \text { where } \mu=0,1
$$

$i s$

$$
j_{2}^{*}\left(s_{t}\right)=\sum_{1 \leq i<j \leq m} \lambda_{i, j}\left(a_{i}, a_{j}\right)+\mu\left(a_{i_{1}}^{m_{i_{1}}} \cdots a_{i_{k}}^{m_{i_{k}}}, \omega\right) .
$$

The last theorem is a direct consequence of Theorem 2.7 and Proposition 2.6. We will now prove Theorem 2.7.

\section{Proof of Theorem 2.7.}

Step 1: We begin to prove that the representation $\rho$ is such that

$$
s_{\rho}=\sum_{1 \leq i<j \leq m}\left(a_{i}, a_{j}^{\lambda_{i, j}}\right)_{L}=\sum_{i=1}^{m-1}\left(a_{i}, a_{i+1}^{\lambda_{i, i+1}} \cdots a_{m}^{\lambda_{i, m}}\right)_{L},
$$

where we obtain the last equality by properties of Galois symbols.

To show it, we prove by induction on $r$ that if $t_{r}=\rho_{1} \hat{\otimes} \cdots \hat{\otimes} \rho_{r}$, then

$$
s_{t_{r}}=\sum_{i=1}^{r}\left(a_{i}, a_{i+1}^{\lambda_{i, i+1}} \cdots a_{m}^{\lambda_{i, m}}\right)_{L}
$$

- If $r=1$,

$$
t_{1}=\rho_{1}=\phi_{1} \hat{\otimes} \phi_{2}^{\lambda_{1,2}} \cdots \phi_{m}^{\lambda_{1, m}}
$$

and

$$
s_{\rho_{1}}=\left(b_{\phi_{1}}, b_{\phi_{2}^{\lambda_{1}, 2} \ldots \phi_{m}^{\lambda_{1, m}}}\right)_{L}=\left(a_{1}, a_{2}^{\lambda_{1,2}} \cdots a_{m}^{\lambda_{1, m}}\right)_{L}
$$

by [7, Proposition 5.5]. Moreover,

$$
b_{\rho_{1}}=a_{1} a_{2}^{\lambda_{1,2}} \cdots a_{m}^{\lambda_{1, m}}
$$

- We suppose the result is true for $r$ and we will compute $s_{t_{r+1}}$. We have, for any $i$, that

$$
s_{\rho_{i}}=\left(a_{i}, a_{i+1}^{\lambda_{i, i+1}} \cdots a_{m}^{\lambda_{i, m}}\right)_{L}+2\left(a_{i} a_{i+1}^{\lambda_{i, i+1}} \cdots a_{m}^{\lambda_{i, m}}, a_{1}^{d_{i, 1}} \cdots a_{m}^{d_{i, m}}\right)_{L},
$$

and

$$
b_{\rho_{i}}=a_{1}^{d_{i, 1}} \cdots a_{i-1}^{d_{i, i-1}} a_{i}^{2+d_{i, i}} a_{i+1}^{2 \lambda_{i, i+1}+d_{i, i+1}} \cdots a_{m}^{2 \lambda_{i, m}+d_{i, m}} .
$$


Lemma 2.10. $b_{t_{r}}=a_{1}^{d_{r+1,1}} \cdots a_{m}^{d_{r+1, m}}$.

By applying the lemma and properties of symbols, we obtain the result:

$$
\begin{aligned}
s_{t_{r+1}}= & s_{t_{r}}+s_{\rho_{r+1}}+\left(b_{t_{r}}, b_{\rho_{r+1}}\right)_{L} \\
= & \sum_{i=1}^{r+1}\left(a_{i}, a_{i+1}^{\lambda_{i, i+1}} \cdots a_{m}^{\lambda_{i, m}}\right)_{L}+\left(a_{r+1}^{2} a_{r+2}^{2 \lambda_{r+1, r+2}} \cdots a_{m}^{2 \lambda_{r+1, m}}, a_{1}^{d_{r+1,1}} \cdots a_{m}^{d_{r+1, m}}\right)_{L} \\
& \quad+\left(a_{1}^{d_{r+1,1}} \cdots a_{m}^{d_{r+1, m}}, a_{r+1}^{2} a_{r+2}^{2 \lambda_{r+1, r+2}} \cdots a_{m}^{2 \lambda_{r+1, m}}\right)_{L} \\
& =\sum_{i=1}^{r+1}\left(a_{i}, a_{i+1}^{\lambda_{i, i+1}} \cdots a_{m}^{\lambda_{i, m}}\right)_{L} .
\end{aligned}
$$

To prove the lemma we prove by induction the formula:

$$
\begin{aligned}
& b_{t_{r}}=a_{1}^{1+d_{2,1}+\cdots+d_{r, 1}} \prod_{2 \leq k<r} a_{k}^{\lambda_{1, k}+2 \lambda_{2, k}+d_{2, k}+\cdots+2 \lambda_{k-1, k}+d_{k-1, k}+2+d_{k, k}+d_{k+1, k}+\cdots d_{r, k}} . \\
& a_{r}^{\lambda_{1, r}+2 \lambda_{2, r}+d_{2, r}+\cdots+2 \lambda_{r-1, r}+d_{r-1, r}+2+d_{r, r}} \cdot \prod_{k>r} a_{k}^{\lambda_{1, k}+2 \lambda_{2, k}+d_{2, k}+2 \lambda_{3, k}+d_{3, k}+\cdots+2 \lambda_{r, k}+d_{r, k}} .
\end{aligned}
$$

It gives the result for the values of the exponents $d_{r+1, k}$.

If $\mu=0$ we have proved the theorem. Now, we suppose $\mu=1$.

Step 2: We compute $s_{\rho \hat{\otimes} \rho^{\prime}}$. By lemma 2.10, $b_{\rho}=a_{1}^{d_{m, m}} \cdots a_{m}^{d_{m, m}}$. Then

$$
s_{\rho \hat{\otimes} \rho^{\prime}}=s_{\rho}+s_{\psi_{i_{1}}^{m_{i_{1}} \ldots \psi_{i_{k}}}}^{m_{i_{k}}}+\left(b_{\rho}, a_{i_{1}}^{m_{i_{1}}} \cdots a_{i_{k}}^{m_{i_{k}}}\right)_{L} .
$$

Step 3: In addition,

$$
s_{\rho^{\prime \prime}}=\left(a_{i_{1}}^{m_{i_{1}}} \cdots a_{i_{k}}^{m_{i_{k}}}, a_{1}^{d_{m, 1}} \cdots a_{m}^{d_{m, m}}\right)_{L},
$$

and then, by properties of symbols,

$$
s_{t}=s_{\rho \hat{\otimes} \rho^{\prime}}+s_{\rho^{\prime \prime}}+\left(b_{\rho \hat{\otimes} \rho^{\prime}}, b_{\rho^{\prime \prime}}\right)=\sum_{1 \leq i<j \leq m} \lambda_{i, j}\left(a_{i}, a_{j}\right)_{L}+\left(a_{i_{1}}^{m_{i_{1}}} \cdots a_{i_{k}}^{m_{i_{k}}}\right) .
$$

By considering the representation of degree 0 associated to the given in Theorem 2.7 following [7, Section 6] and with the method of [7, Section 8] we can compute the solutions of the corresponding embedding problem. 


\section{An example: The exact sequence}

$$
0 \rightarrow \mathbb{Z} / n \mathbb{Z} \rightarrow G \rightarrow \mathbb{Z} / n \mathbb{Z} \times \mathbb{Z} / n \mathbb{Z} \times \mathbb{Z} / n \mathbb{Z} \rightarrow 0
$$

\subsection{Computation of the obstruction}

We consider $n$ odd and $K$ as before and the field extension

$$
L=K\left(\sqrt[n]{a_{1}}, \sqrt[n]{a_{2}}, \sqrt[n]{a_{3}}\right)
$$

such that $\left[K\left(\sqrt{a_{i}}\right): K\right]=n, i=1,2,3$ and $[L: K]=n^{3}$ with

$$
\Gamma=\operatorname{Gal}(L / K) \simeq \mathbb{Z} / n \mathbb{Z} \times \mathbb{Z} / n \mathbb{Z} \times \mathbb{Z} / n \mathbb{Z} .
$$

Let $\sigma_{1}, \sigma_{2}$ and $\sigma_{3}$ be generators of $\Gamma$ determined by $\sigma_{i}\left(\sqrt[n]{a_{j}}\right)=\omega^{\delta_{i j}} \sqrt[n]{a_{j}}$.

Problem 3.1. Let us consider the embedding problem given by

$$
L=K\left(\sqrt[n]{a_{1}}, \sqrt[n]{a_{2}}, \sqrt[n]{a_{3}}\right) / K \text { with } \Gamma=\operatorname{Gal}(L / K) \simeq \mathbb{Z} / n \mathbb{Z} \times \mathbb{Z} / n \mathbb{Z} \times \mathbb{Z} / n \mathbb{Z}
$$

and the exact sequence

$$
(E): \quad 0 \rightarrow \mathbb{Z} / n \mathbb{Z} \stackrel{i}{\rightarrow} G \stackrel{h}{\rightarrow} \Gamma \rightarrow 0
$$

where $G$ is the group

$$
\begin{aligned}
G:=<g_{1}, g_{2}, g_{3} \mid & g_{1}, g_{2} \text { have order } n, g_{3} \text { has order } n^{2}, \\
& g_{1} g_{2}=g_{2} g_{1} g_{3}^{n}, g_{1} g_{3}=g_{3} g_{1}, g_{2} g_{3}=g_{3} g_{2}>,
\end{aligned}
$$

and the morphisms are $h\left(g_{1}\right)=\sigma_{1}, h\left(g_{2}\right)=\sigma_{2}, h\left(g_{3}\right)=\sigma_{3}$ and $i(\lg (\omega))=g_{3}^{n}$.

Remark 3.2. $G$ is a non commutative group of order $n^{4}$ extension of $\Gamma$.

Other groups with these properties are $E_{1} \times \mathbb{Z} / n \mathbb{Z}$ where

$$
E_{1}=<\sigma, \tau \mid \sigma \text { has order } n, \tau \text { has order } n^{2}, \sigma \tau=\tau^{n+1} \sigma>,
$$

and $E_{2} \times \mathbb{Z} / n \mathbb{Z}$ where

$$
E_{2}=<\sigma, \tau, \rho \mid \sigma, \tau, \rho \text { have order } n, \sigma \tau \sigma^{-1} \tau^{-1}=\rho, \sigma \rho=\rho \sigma, \tau \rho=\rho \tau>.
$$

If $n=p$ prime integer, it is the Heisenberg group of degree $p$.

The obstruction to the solvability of the problems given by them is $\left(\omega^{-1} a_{1}, a_{2}\right)$ and $\left(a_{1}, a_{2}\right)$ respectively. These obstructions are computed in [7, Theorems 7.2 and 7.4]). If $n=2$ there are not other noncommutative groups of order $n^{4}([4$, sec. 4$])$. 
Proposition 3.3. The element $\varepsilon \in H^{2}(\Gamma, \mathbb{Z} / n \mathbb{Z})$ corresponding to the exact sequence of the previous problem $(E)$ is $\varepsilon=\left(a_{1}, a_{2}\right)_{L}+\left(a_{3}\right)$.

Proof. We compute the cocycles $a, a^{\prime}, a^{\prime \prime}: \Gamma \times \Gamma \rightarrow \mathbb{Z} / n \mathbb{Z}$ associated to $(E)$, $\left(a_{1}, a_{2}\right)_{L}$ and $\left(a_{3}\right)$ respectively.

The one associated to $(E)$ is

$$
a_{\sigma_{1}^{r} \sigma_{2}^{k} \sigma_{3}^{l}, \sigma_{1}^{\alpha} \sigma_{2}^{b} \sigma_{3}^{c}}= \begin{cases}-k \alpha & \text { if } l+c<n \\ -k \alpha+1 & \text { if } l+c \geq n .\end{cases}
$$

The cocycle associated to $\left(a_{1}, a_{2}\right)_{L}=-\left(a_{2}, a_{1}\right)_{L}$ is

$$
a_{\sigma_{1}^{r} \sigma_{2}^{k} \sigma_{3}^{l}, \sigma_{1}^{\alpha} \sigma_{2}^{b} \sigma_{3}^{c}}^{\prime}=-k \alpha,
$$

and the cocycle associated to $\left(a_{3}\right)$ is

$$
a_{\sigma_{1}^{\prime} \sigma_{2}^{k} \sigma_{3}^{l}, \sigma_{1}^{\alpha} \sigma_{2}^{b} \sigma_{3}^{c}}^{\prime \prime}= \begin{cases}0 & \text { if } l+c<n \\ 1 & \text { if } l+c \geq n .\end{cases}
$$

So, $a=a^{\prime}+a^{\prime \prime}$ and we get the result.

Applying Theorem 2.7, the representation corresponding to the embedding problem 3.1 is

$$
t=\rho \hat{\otimes}\left(\rho^{\prime} \hat{\otimes} \rho^{\prime \prime}\right)
$$

where $\rho=\rho_{1} \hat{\otimes} \rho_{2}$ for $\rho_{1}=\phi_{1} \hat{\otimes} \phi_{2}$ and $\rho_{2}=\phi_{2} \hat{\otimes} \phi_{2} \hat{\otimes} \phi_{1} \phi_{2}, \rho^{\prime}=\psi_{3}$ and $\rho^{\prime \prime}=\phi_{3} \hat{\otimes} \phi_{1}^{2} \phi_{2}^{4}$.

Writing the representation in terms of generalized Clifford algebras and morphism it is

$$
C_{t}=C\left(e_{1}, e_{2}, e_{3}, e_{4}, e_{5}, v, e_{6}, e_{7}\right) \text { with } e_{i}^{n}=1 \text { for } i=1, \ldots, 7 \text {, and } v^{n}=\omega
$$

and

$$
\begin{aligned}
t: \Gamma & \longrightarrow \operatorname{Autgr}\left(C_{t}\right) \\
\sigma_{1} & \longrightarrow \varphi\left(e_{1} e_{5} e_{7}^{2}\right) \\
\sigma_{2} & \longrightarrow \varphi\left(e_{2} e_{3} e_{4} e_{5} e_{7}^{4}\right) \\
\sigma_{3} & \longrightarrow \varphi\left(v e_{6}\right) .
\end{aligned}
$$

By putting $u_{1}:=e_{1} e_{5} e_{7}^{2}, u_{2}:=e_{2} e_{3} e_{4} e_{5} e_{7}^{4}$ and $u_{3}:=v e_{6}$, they satisfy the next relations

$$
\begin{aligned}
& u_{1}^{k}=\omega^{-5 \frac{k(k-1)}{2}} e_{1}^{k} e_{5}^{k} e_{7}^{2 k}, \quad \text { so } u_{1}^{n}=1, \\
& u_{2}^{k}=\omega^{-22 \frac{k(k-1)}{2}} e_{2}^{k} e_{3}^{k} e_{4}^{k} e_{5}^{k} e_{7}^{4 k}, \quad \text { so } u_{2}^{n}=1, \\
& u_{3}^{k}=\omega^{-\frac{k(k-1)}{2}} v^{k} e_{6}^{k}=\omega, \quad \text { so } u_{3}^{n}=\omega
\end{aligned}
$$

and moreover the relations

$$
u_{1} u_{2}=\omega u_{2} u_{1}, \quad u_{1} u_{3}=u_{3} u_{1}, \quad \text { and } \quad u_{2} u_{3}=u_{3} u_{2} .
$$

Then, we have checked again that $\varepsilon=s_{t}$. 
The twisted algebra by the representation $t$ is

$$
\begin{aligned}
\mathfrak{C}_{t}= & C\left(e_{1} \otimes \sqrt[n]{a_{1}^{3} a_{2}^{8} a_{3}^{2}}, e_{2} \otimes \sqrt[n]{a_{1}^{2} a_{2}^{7} a_{3}^{2}}, e_{3} \otimes \sqrt[n]{a_{1}^{2} a_{2}^{5} a_{3}^{2}}, e_{4} \otimes \sqrt[n]{a_{1}^{2} a_{2}^{3} a_{3}^{2}},\right. \\
& \left.e_{5} \otimes \sqrt[n]{a_{1} a_{2} a_{3}^{2}}, v \otimes \sqrt[n]{a_{3}}, e_{6} \otimes \sqrt[n]{a_{3}^{-1}}, e_{7} \otimes \sqrt[n]{a_{1}^{-2} a_{2}^{-4} a_{3}^{-2}}\right) \\
= & C\left(a_{1}^{3} a_{2}^{8} a_{3}^{2}, a_{1}^{2} a_{2}^{7} a_{3}^{2}, a_{1}^{2} a_{2}^{5} a_{3}^{2}, a_{1}^{2} a_{2}^{3} a_{3}^{2}, a_{1} a_{2} a_{3}^{2}, \omega a_{3}, a_{3}^{-1}, a_{1}^{-2} a_{2}^{-4} a_{3}^{-2}\right)
\end{aligned}
$$

because the generating elements are fixed by the action $t \otimes$ gal of $\Gamma$. The element $b_{t}=a_{1}^{4} a_{2}^{8} a_{3}^{2}$.

Corollary 3.4. The obstruction to the solvability is

$$
j_{2}^{*}\left(s_{t}\right)=\left[\mathfrak{C}_{t}\right]=\left(a_{1}, a_{2}\right)+\left(a_{3}, \omega\right) .
$$

For instance, for $n=3, K=\mathbb{Q}(\omega)$ (where $\omega$ is a third root of unity) and $a_{1}=2, a_{2}=5$ and $a_{3}=3$ the embedding problem is solvable because $(2,5)=0,(3, \omega)=0$ and therefore $j_{2}^{*}\left(s_{t}\right)$.

The representation of degree 0 corresponding to $t$ is

$$
\begin{aligned}
t_{0}: \Gamma & \longrightarrow \operatorname{Autgr}\left(C_{t}\right) \\
\sigma_{1} & \longrightarrow \varphi\left(e_{1} e_{5} e_{7}^{2} e_{8}^{-4}\right) \\
\sigma_{2} & \longrightarrow \varphi\left(e_{2} e_{3} e_{4} e_{5} e_{7}^{4} e_{8}^{-8}\right) \\
\sigma_{3} & \longrightarrow \varphi\left(v e_{6} e_{8}^{-2}\right)
\end{aligned}
$$

where

$C_{t_{0}}=C\left(e_{1}, e_{2}, e_{3}, e_{4}, e_{5}, v, e_{6}, e_{7}, e_{8}\right)$ with $e_{i}^{n}=1$ for $i=1, \ldots, 8$, and $v^{n}=\omega$.

The twisted algebra by $t_{0}$ is

$$
\begin{aligned}
& \mathfrak{C}_{t_{0}}=C\left(e_{1} \otimes \sqrt[n]{a_{1}^{-1}}, e_{2} \otimes \sqrt[n]{a_{1}^{-2} a_{2}^{-1}}, e_{3} \otimes \sqrt[n]{a_{1}^{-2} a_{2}^{-3}}, e_{4} \otimes \sqrt[n]{a_{1}^{-2} a_{2}^{-5}},\right. \\
& e_{5} \otimes \sqrt[n]{a_{1}^{-3} a_{2}^{-7}}, v \otimes \sqrt[n]{a_{1}^{-4} a_{2}^{-8} a_{3}^{-1}}, e_{6} \otimes \sqrt[n]{a_{1}^{-4} a_{2}^{-8} a_{3}^{-3}}, \\
& \left.e_{7} \otimes \sqrt[n]{a_{1}^{-6} a_{2}^{-12} a_{3}^{-4}}, e_{8} \otimes \sqrt[n]{a_{1}^{-4} a_{2}^{-8} a_{3}^{-2}}\right) \\
& =C\left(a_{1}^{-1}, a_{1}^{-2} a_{2}^{-1}, a_{1}^{-2} a_{2}^{-3}, a_{1}^{-2} a_{2}^{-5}, a_{1}^{-3} a_{2}^{-7}, \omega a_{1}^{-4} a_{2}^{-8} a_{3}^{-1}, a_{1}^{-4} a_{2}^{-8} a_{3}^{-3}\right. \text {, } \\
& \left.a_{1}^{-6} a_{2}^{-12} a_{3}^{-4}, a_{1}^{-4} a_{2}^{-8} a_{3}^{-2}\right) \text {. }
\end{aligned}
$$

To find the solution of the embedding problem it is only necessary determine an isomorphism over $K$ from $C_{t_{0}}$ into $\mathfrak{C}_{t_{0}}$ and compute the elements $z \in \mathfrak{C}_{t_{0}}$ and $\gamma \in L$ using the method given in [7, Section 8].

Remark 3.5. Given a embedding problem may be there is a representation simpler than the given in Theorem 2.7, but this theorem assure the existence of at least one. 
In order to compute an element $\gamma \in L$ such that $L(\sqrt[n]{\gamma})$ is a solution of the embedding problem and to simplify the computations, we will consider another simpler representation $T$ of degree 0 of $\Gamma$ which allows us to determine the isomorphism over $K$ of $C_{T}$ into $\mathfrak{C}_{T}$ more easily and then, compute the elements $z$ and $\gamma$.

We consider the representation $(C, T)$ where

$$
C=C\left(e_{1}, e_{2}, e_{3}, e_{4}\right)=C\left(1,1,1, \omega^{-1}\right)
$$

and the representation

$$
T: \Gamma \rightarrow O(C), \quad \sigma_{1} \mapsto \varphi\left(e_{1} e_{2}^{-1}\right), \sigma_{2} \mapsto \varphi\left(e_{1} e_{3}^{-1}\right), \sigma_{3} \mapsto \varphi\left(e_{1} e_{2}^{-1} e_{3} e_{4}^{-1}\right) .
$$

The twisted algebra by $T$ is

$$
\mathfrak{C}=C\left(v_{1}, v_{2}, v_{3}, v_{4}\right)=C\left(a_{1}^{-1} a_{2}^{-1} a_{3}^{-1}, a_{1}^{-1} a_{2}^{-2} a_{3}^{-1}, a_{2}^{-1} a_{3}^{-1}, \omega^{-1} a_{3}^{-1}\right)
$$

because the elements

$$
\begin{aligned}
& v_{1}=e_{1} \otimes \sqrt[n]{a_{1}^{-1} a_{2}^{-1} a_{3}^{-1}}, \\
& v_{2}=e_{2} \otimes \sqrt[n]{a_{1}^{-1} a_{2}^{-2} a_{3}^{-1}} \\
& v_{3}=e_{3} \otimes \sqrt[n]{a_{2}^{-1} a_{3}^{-1}} \\
& v_{4}=e_{4} \otimes \sqrt[n]{a_{3}^{-1}},
\end{aligned}
$$

are fixed by the action $T \otimes$ gal. Clearly this representation is of degree 0 and it is straightforward to check that $\varepsilon=s_{T}$.

The obstruction to the solvability of the considered embedding problem is $\left(a_{1}, a_{2}\right)+\left(a_{3}, \omega\right)$. We suppose the problem is solvable, that is $\left(a_{1}, a_{2}\right)+$ $\left(a_{3}, \omega\right)=0$, and we want to find an element $\gamma \in L^{*}$ such that $M=L(\sqrt[n]{\gamma})$ is a solution.

Moreover, to simplify the computations we suppose, that the symbols

$$
\left(a_{1}, a_{2}\right)=0,\left(a_{3}, \omega\right)=0 \text { and }\left(a_{2}, a_{3}\right)=0 .
$$

For the values $a_{1}=2, a_{2}=5, a_{3}=3$ and $n=3$ it is true.

\subsection{The isomorphism $g: C_{L} \rightarrow \mathfrak{C}_{L}$ over $L$}

Proposition 3.6. The isomorphism $g: C_{L} \rightarrow \mathfrak{C}_{L}$ given by:

$$
e_{1} \mapsto \sqrt[n]{a_{1} a_{2} a_{3}} v_{1}, \quad e_{2} \mapsto \sqrt[n]{a_{1} a_{2}^{2} a_{3}} v_{2}, \quad e_{3} \mapsto \sqrt[n]{a_{2} a_{3}} v_{3}, \quad e_{4} \mapsto \sqrt[n]{a_{3}} v_{4}
$$

is a graded isomorphism which satisfies $g^{-1} g^{\tau}=T(\tau)$ for each $\tau \in \Gamma$.

The proof is a simple computation. 
We express the isomorphism $g$ as the composition of the three isomorphisms $g_{1}, g_{2}$ and $g_{3}$

$$
C_{L} \stackrel{g_{1}}{\simeq} C_{2}=C\left(1,1, a_{3}^{-1}, \omega^{-1} a_{3}^{-1}\right)_{L} \stackrel{g_{2}}{\simeq} C_{3}=C\left(1, a_{2}^{-1}, a_{2}^{-1} a_{3}^{-1}, \omega^{-1} a_{3}^{-1}\right)_{L} \stackrel{g_{3}}{\simeq} \mathfrak{C}_{L}
$$

given by

$$
\begin{array}{lllll}
g_{1}\left(e_{1}\right)=u_{1}, & g_{1}\left(e_{2}\right)=u_{2}, & g_{1}\left(e_{3}\right)=\sqrt[n]{a_{3}} u_{3}, & & g_{1}\left(e_{4}\right)=\sqrt[n]{a_{3}} u_{4} \\
g_{2}\left(u_{1}\right)=w_{1}, & g_{2}\left(u_{2}\right)=\sqrt[n]{a_{2}} w_{2}, & g_{2}\left(u_{3}\right)=\sqrt[n]{a_{2}} w_{3}, & & g_{2}\left(u_{4}\right)=w_{4} \\
g_{3}\left(w_{1}\right)=\sqrt[n]{a} v_{1}, & g_{3}\left(w_{2}\right)=\sqrt[n]{a} v_{2}, & g_{3}\left(w_{3}\right)=v_{3}, & & g_{3}\left(w_{4}\right)=v_{4}
\end{array}
$$

where $a=a_{1} a_{2} a_{3}$ and the elements $u_{1}, u_{2}, u_{3}, u_{4}$ and $w_{1}, w_{2}, w_{3}, w_{4}$ generate the algebras $C_{2}=C\left(1,1, a_{3}^{-1}, \omega^{-1} a_{3}^{-1}\right)$ and $C_{3}=C\left(1, a_{2}^{-1}, a_{2}^{-1} a_{3}^{-1}, \omega^{-1} a_{3}^{-1}\right)$ respectively.

Clearly $g=g_{3} \circ g_{2} \circ g_{1}$.

\subsection{The isomorphism $f: C \rightarrow \mathfrak{C}$ over $K$}

We know that there exists an isomorphism $f$ defined over $K$ from $C$ into $\mathfrak{C}$. To determine $f$, we express this isomorphism in terms of three easier isomorphisms between generalized Clifford algebras generated by two elements. We consider the isomorphisms

$$
C \stackrel{f_{1}}{\simeq} C_{2}=C\left(1,1, a_{3}^{-1}, \omega^{-1} a_{3}^{-1}\right) \stackrel{f_{2}}{\simeq} C_{3}=C\left(1, a_{2}^{-1}, a_{2}^{-1} a_{3}^{-1}, \omega^{-1} a_{3}^{-1}\right) \stackrel{f_{3}}{\simeq} \mathfrak{C}
$$

where they are obtained from the isomorphism $C(1, \beta) \simeq C(\alpha, \alpha \beta)$, valid if the symbol $(\alpha, \beta)=0, \alpha, \beta \in K$. For a generalized Clifford algebra $C=C\left(\nu_{1}, \nu_{2}\right)$, the vector subspace of the elements of degree $i$ of $C$ is denoted by $C_{i}$. We consider the following basis of the subspaces $C_{i}$ for $i=0, \ldots, n-1$ :

$$
\omega^{c(k)-(i-1) k} e_{1}^{n+i-k} e_{2}^{k}, \quad k=0, \ldots, n-1
$$

where $c(0)=0$ and $c(k)=k x+\frac{k(k-1)}{2}$ for $k>0$, where $x$ is an arbitrary integer.

Lemma 3.7. We consider the algebras $C\left(\nu_{1}, \nu_{2}\right)=C(1, \beta)$ and $C\left(v_{1}, v_{2}\right)=$ $C(\alpha, \alpha \beta)$. If the Galois symbol $(\alpha, \beta)=0$, there exists an isomorphism $F: C(1, \beta) \simeq C(\alpha, \alpha \beta)$ defined over $K$ given by:

$\nu_{1} \mapsto \frac{1}{\alpha^{2}} \sum_{k=0}^{n-1} y_{k} \omega^{c(k)} v_{1}^{n+1-k} v_{2}^{k}, \nu_{2} \mapsto \frac{1}{\alpha^{2}} \beta y_{n-1} v^{n+1}+\sum_{k=1}^{n-1} y_{k-1} \omega^{c(k)} v_{1}^{n+1-k} v_{2}^{k}$,

where $y_{0}+y_{1} \sqrt[n]{\beta}+\cdots+y_{n-1} \sqrt[n]{\beta^{n-1}}$ is an element of $K(\sqrt[n]{\beta})$ with norm $\alpha^{n-1}$.

The proof of this lemma is similar to the proof of [7, Theorems 10.2 and 11.5]. 
From the previous lemma, we can compute the isomorphisms $f_{1}, f_{2}$ and $f_{3}$ over $K$. For the first one the values are $\alpha=a_{3}^{-1}$ and $\beta=\omega^{-1}$ and the isomorphism exists because we have supposed that the symbol

$$
\left(a_{3}^{-1}, \omega^{-1}\right)=\left(a_{3}, \omega\right)=0 .
$$

For $f_{2}$, the values are $\alpha=a_{2}^{-1}$ and $\beta=a_{3}^{-1}$ and we have supposed that the symbol $\left(a_{2}, a_{3}\right)=0$.

For the last one $\alpha=a_{1}^{-1} a_{2}^{-1} a_{3}^{-1}$ and $\beta=a_{2}^{-1}$. The symbol

$$
\left(a_{1}^{-1} a_{2}^{-1} a_{3}^{-1}, a_{2}^{-1}\right)=\left(a_{1} a_{2} a_{3}, a_{2}\right)=\left(a_{1}, a_{2}\right)+\left(a_{2}, a_{2}\right)+\left(a_{3}, a_{2}\right)=0
$$

because we have supposed $\left(a_{1}, a_{2}\right)=0=\left(a_{2}, a_{3}\right)$ and $\left(a_{2}, a_{2}\right)=0$ since $n$ is odd.

Thus, we have the isomorphism $f$ as composition of these isomorphisms.

In particular, for $n=3$ and $a_{1}=2, a_{2}=5, a_{3}=3$ we have found the elements to determine these isomorphisms:

For $f_{1}, \alpha=\frac{1}{3}, \beta=\omega^{-1}$ and the element

$$
\frac{1}{3}\left(2-\sqrt[3]{\beta}-\omega \sqrt[3]{\beta^{2}}\right) \in K(\sqrt[3]{\beta})
$$

has norm $\frac{1}{9}$.

For $f_{2}, \alpha=\frac{1}{5}, \beta=\frac{1}{3}$ and the element

$$
\frac{1}{25}\left(28+36 \sqrt[3]{\beta}+57 \sqrt[3]{\beta^{2}}\right) \in K(\sqrt[3]{\beta})
$$

has norm $\frac{1}{25}$.

For $f_{3}, \alpha=\frac{1}{30}, \beta=\frac{1}{5}$ and the element

$$
\frac{1}{12}\left(1+\sqrt[3]{\beta}-3 \sqrt[3]{\beta^{2}}\right) \in K(\sqrt[3]{\beta})
$$

has norm $\frac{1}{900}$.

The isomorphisms are then:

$$
\begin{aligned}
f_{1}: C \rightarrow C_{2}, & e_{1} \mapsto u_{1}, \quad e_{2} \mapsto u_{2}, e_{3} \mapsto 2 u_{3}-u_{4}-3 \omega^{2} u_{3}^{2} u_{4}^{2}, \\
& e_{4} \mapsto-u_{3}+2 u_{4}-3 u_{3}^{2} u_{4}^{2}, \\
f_{2}: C_{2} \rightarrow C_{3}, \quad & u_{1} \mapsto w_{1}, \quad u_{2} \mapsto 5\left(28 w_{2}+36 w_{3}+285 w_{2}^{2} w_{3}^{2}\right), \\
& u_{3} \mapsto 5\left(19 w_{2}+28 w_{3}+180 w_{2}^{2} w_{3}^{2}\right), \quad u_{4} \mapsto w_{4}, \\
f_{3}: C_{3} \rightarrow \mathfrak{C}, & w_{1} \mapsto \frac{75}{30}\left(v_{1}+v_{2}-90 v_{1}^{2} v_{2}^{2}\right), \\
& w_{2} \mapsto \frac{-3}{2} v_{1}+\frac{75}{30}\left(v_{2}+30 v_{1}^{2} v_{2}^{2}\right), \quad w_{3} \mapsto v_{3} \quad w_{4} \mapsto v_{4} .
\end{aligned}
$$




\subsection{The element $z$}

From the isomorphisms $f_{1}, g_{1}, f_{2}, g_{2}$ and $f_{3}, g_{3}$, following [7, Theorem 8.2] and its proof, we can compute elements $z_{1} \in\left(C_{2}\right)_{L}, z_{2} \in\left(C_{3}\right)_{L}$ and $z_{3} \in \mathfrak{C}_{L}$ such that $g_{i}(x)=z_{i} f_{i}(x) z_{i}^{-1}$ for $i=1,2,3$ and $x$ any element of the corresponding Clifford algebra. The element $z=g_{3}\left(g_{2}\left(z_{1}\right) z_{2}\right) z_{3} \in \mathfrak{C}_{L}$ satisfies $g(x)=z f(x) z^{-1}$ for all $x \in C_{L}$.

We compute now the elements $z_{1}, z_{2}$ and $z_{3}$. As $f_{1}\left(e_{1}\right)=g_{1}\left(e_{1}\right)$ and $f_{1}\left(e_{2}\right)=g_{1}\left(e_{2}\right)$, we can express

$$
z_{1}=\sum_{\epsilon_{i} \in\{0, \ldots, n-1\}} g_{1}\left(e_{3}\right)^{\epsilon_{3}} g_{1}\left(e_{4}\right)^{\epsilon_{4}} f_{1}\left(e_{4}\right)^{-\epsilon_{4}} f_{1}\left(e_{3}\right)^{-\epsilon_{3}},
$$

as $f_{2}\left(u_{1}\right)=g_{2}\left(u_{1}\right)$ and $f_{2}\left(u_{4}\right)=g_{2}\left(u_{4}\right)$,

$$
z_{2}=\sum_{\epsilon_{i} \in\{0, \ldots, n-1\}} g_{2}\left(u_{2}\right)^{\epsilon_{2}} g_{2}\left(u_{3}\right)^{\epsilon_{3}} f_{2}\left(u_{3}\right)^{-\epsilon_{3}} f_{2}\left(u_{2}\right)^{-\epsilon_{2}}
$$

and, as $f_{3}\left(w_{3}\right)=g_{3}\left(w_{3}\right)$ and $f_{3}\left(w_{4}\right)=g_{3}\left(w_{4}\right)$,

$$
z_{3}=\sum_{\epsilon_{i} \in\{0, \ldots, n-1\}} g_{3}\left(w_{1}\right)^{\epsilon_{1}} g_{3}\left(w_{2}\right)^{\epsilon_{2}} f_{3}\left(w_{2}\right)^{-\epsilon_{2}} f_{3}\left(u_{1}\right)^{-\epsilon_{1}}
$$

We compute these elements using the package $n$-Clifford.

For instance, for $n=3$ the coordinates of the elements $z_{1}, z_{2}$ and $z_{3}$ in the fixed basis are:

$$
\begin{gathered}
z_{1}=\left\{\omega y_{0}^{2}+\frac{\omega y_{0}}{a_{3}^{2 / 3}}+\frac{\omega}{a_{3}^{4 / 3}}-y_{1} y_{2}, y_{2}^{2}+\left(\frac{1}{a_{3}^{2 / 3}}-\omega y_{0}\right) y_{1},\right. \\
\left.\frac{1}{a_{3}^{2 / 3}}\left(a_{3}^{2 / 3} \omega y_{1}^{2}-\left(a_{3}^{2 / 3} y_{0} \omega+\omega+1\right) y_{2}\right)\right\}
\end{gathered}
$$

where $y_{0}+y_{1} \sqrt[3]{\omega^{-1}}+y_{2} \sqrt[3]{\omega^{-2}} \in K\left(\sqrt[3]{\omega^{-1}}\right)$ such that $N_{K\left(\sqrt[3]{\omega^{-1}}\right) / K}(y)=a_{3}^{-2}$,

$$
\begin{aligned}
z_{2}= & \left\{a_{3} y_{0}^{2}+\frac{a_{3} y_{0}}{a_{2}^{2 / 3}}+\frac{a_{3}}{a_{2}^{4 / 3}}-y_{1} y_{2}, \frac{1}{a_{2}^{2 / 3}}\left(a_{2}^{2 / 3} y_{2}^{2}-a_{3}\left(\omega+a_{2}^{2 / 3} y_{0}+1\right) y_{1}\right),\right. \\
& \left.a_{3}\left(y_{1}^{2}+\left(\frac{\omega}{a_{2}^{2 / 3}}-y_{0}\right) y_{2}\right)\right\}
\end{aligned}
$$

where $y_{0}+y_{1} \sqrt[3]{a_{3}^{-1}}+y_{2} \sqrt[3]{a_{3}^{-2}} \in K\left(\sqrt[3]{a_{3}^{-1}}\right)$ such that $N_{K\left(\sqrt[3]{a_{3}^{-1}}\right) / K}(y)=a_{2}^{-2}$ 
and

$$
\begin{aligned}
z_{3}= & \left\{\left(a_{1} a_{2} a_{3}\right)^{4 / 3} y_{0}^{2}+\left(\begin{array}{lll}
a_{1} & a_{2} & a_{3}
\end{array}\right)^{2 / 3} y_{0}-a_{1} a_{3} \sqrt[3]{a_{1} a_{2} a_{3}} y_{1} y_{2}+1\right. \\
& \sqrt[3]{a_{1}^{2} a_{2} a_{3}^{2}}\left(\sqrt[3]{a_{1}^{2} a_{3}^{2}} y_{2}^{2}-\sqrt[3]{a_{2}}\left(\omega+\sqrt[3]{a_{1}^{2} a_{2}^{2} a_{3}^{2}} y_{0}+1\right) y_{1}\right) \\
& \left.\left(a_{1} a_{2} a_{3}\right)^{4 / 3} y_{1}^{2}+\sqrt[3]{a_{1}^{2} a_{2}^{2} a_{3}^{2}}\left(\omega-\sqrt[3]{a_{1}^{2} a_{2}^{2} a_{3}^{2}} y_{0}\right) y_{2}\right\}
\end{aligned}
$$

where $y_{0}+y_{1} \sqrt[3]{a_{2}^{-1}}+y_{2} \sqrt[3]{a_{2}^{-2}} \in K\left(\sqrt[3]{a_{2}^{-1}}\right)$ such that

$$
N_{K\left(\sqrt[3]{a_{2}^{-1}}\right) / K}(y)=\left(a_{1} a_{2} a_{3}\right)^{-1} .
$$

For $a_{1}=2, a_{2}=5, a_{3}=3$ the values are:

$$
\begin{aligned}
& z_{1}=\{\omega(3+3 \sqrt[3]{3}+2 \sqrt[3]{9}), \sqrt[3]{3}(\omega-1-\sqrt[3]{3}), \sqrt[3]{3}(-\omega-2-\sqrt[3]{3})\} \\
& z_{2}=\left\{25+20 \sqrt[3]{5}+28 \sqrt[3]{25}, 3\left(5 \sqrt[3]{5}+12 \omega^{2} \sqrt[3]{25}\right), 3 \sqrt[3]{5}(-20+19 \omega \sqrt[3]{5})\right\} \\
& z_{3}=\left\{12+4 \sqrt[3]{30}+\sqrt[3]{900}, \sqrt[3]{30}\left(2+\omega^{2} \sqrt[3]{30}\right), \sqrt[3]{30}(10-3 \omega \sqrt[3]{30})\right\}
\end{aligned}
$$

\subsection{The element $\gamma$}

We look now for an element $\gamma \in L$ such that $M=L(\sqrt[n]{\gamma})$ is a solution to the embedding problem. We compute $\gamma=\gamma_{1} \gamma_{2} \gamma_{3}$ where $\gamma_{1} \in L$ is the element corresponding to $z_{1}, \gamma_{2} \in L$ is the element corresponding to $z_{2}$ and $\gamma_{3} \in L$ is the element corresponding to $z_{3}$. We know (see the proof of [7, Theorem 8.2]) that the element $b_{\sigma}=z z^{-\sigma} g\left(u_{\sigma}\right) \in L$ (where the element $u_{\sigma} \in F\left(C_{L}\right)$ is such that $\left.\varphi_{C_{L}}\left(u_{\sigma}\right)=t(\sigma)\right)$ satisfies $b_{\sigma} b_{\tau}^{\sigma}=a_{\sigma, \tau} b_{\sigma \tau}$ (where the cocycle $a_{\sigma, \tau}$ represents $\varepsilon$ ) and the element $\gamma$ that satisfies $\gamma^{\sigma} \gamma^{-1}=b_{\sigma}^{n}$ gives a solution to the embedding problem.

We consider the associated representations to $T, T_{i}$ such that $g_{i}^{-1} g_{i}^{\sigma}=$ $T_{i}(\sigma)$ for $i=1,2,3$. They are:

$$
\begin{array}{lll}
T_{1}: \Gamma \rightarrow O(C), & \sigma_{1} \mapsto I d, \quad \sigma_{2} \mapsto I d, \quad \sigma_{3} \mapsto \varphi_{C}\left(e_{3}^{-1} e_{4}\right), \\
T_{2}: \Gamma \rightarrow O\left(C_{2}\right), & \sigma_{1} \mapsto I d, \quad \sigma_{2} \mapsto \varphi_{C_{2}}\left(\sqrt[n]{a_{3}^{-1}} u_{2} u_{3}^{-1}\right), \quad \sigma_{3} \mapsto I d, \\
T_{3}: \Gamma \rightarrow O\left(C_{3}\right), & \sigma_{1} \mapsto \varphi_{C_{3}}\left(\sqrt[n]{a_{2}^{-1}} w_{1} w_{2}^{-1}\right), \sigma_{2} \mapsto \varphi_{C_{3}}\left(\sqrt[n]{a_{2}^{-1}} w_{1} w_{2}^{-1}\right), \\
& \sigma_{3} \mapsto \varphi_{C_{3}}\left(\sqrt[n]{a_{2}^{-1}} w_{1} w_{2}^{-1}\right) .
\end{array}
$$

For $T_{1}$, we consider the elements

$$
\begin{gathered}
M_{\sigma_{1}}^{T_{1}}=M_{\sigma_{2}}^{T_{1}}=1, M_{\sigma_{3}}^{T_{1}}=g_{1}\left(e_{3} e_{4}^{-1}\right)=u_{3} u_{4}^{-1} \\
N_{\sigma_{1}}^{T_{1}}=N_{\sigma_{2}}^{T_{1}}=N\left(M_{\sigma_{1}}^{T_{1}}\right)=1, N_{\sigma_{3}}^{T_{1}}=N\left(M_{\sigma_{3}}^{T_{1}}\right)=\omega .
\end{gathered}
$$


For $\sigma \in \Gamma$, we consider the corresponding

$$
M_{\sigma}^{T_{1}}=g_{1}\left(u_{\sigma}^{T_{1}}\right), \quad N_{\sigma}^{T_{1}}=N\left(M_{\sigma}^{T_{1}}\right) \quad \text { and } \quad B_{\sigma}^{T_{1}}=z_{1} z_{1}^{-\sigma} M_{\sigma}^{T_{1}} \in L .
$$

They satisfy $N\left(z_{1}\right)=\left(B_{\sigma}^{T_{1}}\right)^{n}\left(N_{\sigma}^{T_{1}}\right)^{-1} N\left(z_{1}\right)^{\sigma}$. Let $\alpha_{1}$ be a coordinate of $N\left(z_{1}\right)$. The element $\eta_{1}=\sqrt[n]{a_{3}}$ satisfies $\eta_{1}^{-\sigma} \eta_{1}=\left(N_{\sigma}^{T_{1}}\right)^{-1}$. Then, the element $\gamma_{1}=\eta_{1} \alpha_{1}^{-1}$ satisfies the relation $\gamma_{1}^{\sigma}=\left(B_{\sigma}^{T_{1}}\right)^{n} \gamma_{1}$ for each $\sigma \in \Gamma$.

Similarly, we consider for $T_{2}$,

$$
\begin{gathered}
M_{\sigma_{1}}^{T_{2}}=M_{\sigma_{3}}^{T_{2}}=1, M_{\sigma_{2}}^{T_{2}}=g_{2}\left(\sqrt[n]{a_{3}^{-1}} u_{2} u_{3}^{-1}\right)=\sqrt[n]{a_{3}^{-1}} w_{2} w_{3}^{-1}, M_{\sigma}^{T_{2}}=g_{2}\left(u_{\sigma}^{T_{2}}\right), \\
N_{\sigma}^{T_{2}}=N\left(M_{\sigma}^{T 2}\right)=1 \text { and } B_{\sigma}^{T_{2}}=z_{2} z_{2}^{-\sigma} M_{\sigma}^{T_{2}} \in L, \forall \sigma \in \Gamma
\end{gathered}
$$

They satisfy $N\left(z_{2}\right)=\left(B_{\sigma}^{T_{2}}\right)^{n}\left(N_{\sigma}^{T_{2}}\right)^{-1} N\left(z_{2}\right)^{\sigma}$. In this case $\eta_{2}=1$ and, if $\alpha_{2}$ is a coordinate of $N\left(z_{2}\right)$, the element $\gamma_{2}=\alpha_{2}^{-1}$ satisfies the relation $\gamma_{2}^{\sigma}=\left(B_{\sigma}^{T_{2}}\right)^{n} \gamma_{2}$ for each $\sigma \in \Gamma$.

In the same way, we consider for $T_{3}$,

$$
\begin{gathered}
M_{\sigma_{1}}^{T_{3}}=M_{\sigma_{3}}^{T_{3}}=M_{\sigma_{3}}^{T_{3}}=g_{3}\left(\sqrt[n]{a_{2}^{-1}} w_{1} w_{2}^{-1}\right)=\sqrt[n]{a_{2}^{-1}} v_{1} v_{2}^{-1}, M_{\sigma}^{T_{3}}=g_{3}\left(u_{\sigma}^{T_{3}}\right), \\
N_{\sigma}^{T_{3}}=N\left(M_{\sigma}^{T_{3}}\right)=1 \text { and } B_{\sigma}^{T_{3}}=z_{3} z_{3}^{-\sigma} M_{\sigma}^{T_{3}} \in L, \forall \sigma \in \Gamma .
\end{gathered}
$$

We have $N\left(z_{3}\right)=\left(B_{\sigma}^{T_{3}}\right)^{n}\left(N_{\sigma}^{T_{3}}\right)^{-1} N\left(z_{3}\right)^{\sigma}$. Now $\eta_{3}=1$ and $\gamma_{3}=\alpha_{3}^{-1}$ (where $\alpha_{3}$ is a coordinate of $N\left(z_{3}\right)$ ) satisfies the relation $\gamma_{3}^{\sigma}=\left(B_{\sigma}^{T_{3}}\right)^{n} \gamma_{3}$ for each $\sigma \in \Gamma$.

It should be noted that for the representation $T$, the elements

$$
m_{\sigma}=g\left(u_{\sigma}\right)=g_{3}\left(g_{2}\left(M_{\sigma}^{T_{1}}\right) M_{\sigma}^{T_{2}}\right) M_{\sigma}^{T_{3}} \nu_{\sigma}
$$

where $\nu_{\sigma} \in \mu_{n}$. It is not difficult to prove that $b_{\sigma}=B_{\sigma}^{T_{1}} B_{\sigma}^{T_{2}} B_{\sigma}^{T_{3}} \nu_{\sigma}^{\prime}\left(\nu_{\sigma}^{\prime} \in \mu_{n}\right)$, and, therefore, $b_{\sigma}^{n}=\left(B_{\sigma}^{T_{1}} B_{\sigma}^{T_{2}} B_{\sigma}^{T_{3}}\right)^{n}$.

Then, the element $\gamma=\gamma_{1} \gamma_{2} \gamma_{3}$ satisfies $\gamma^{\sigma}=b_{\sigma}^{n} \gamma$ and $M=L(\sqrt[n]{\gamma})$ is a solution to the embedding problem.

The general expression of the elements $\gamma_{1}, \gamma_{2}$ and $\gamma_{3}$ for $n=3$ is:

$$
\begin{aligned}
\gamma_{1}= & \frac{1}{a_{3}^{19 / 3}}\left(3 \left(a_{3}^{4} \omega y_{1}^{6}-a_{3}^{10 / 3}(1+2 \omega) y_{2} y_{1}^{4}-3 a_{3}^{4} \omega y_{0} y_{2} y_{1}^{4}+a_{3}^{4} y_{2}^{3} y_{1}^{3}+\right.\right. \\
& 3 a_{3}^{2} y_{1}^{3}+a_{3}^{10 / 3} y_{0}^{2} y_{1}^{3}+2 a_{3}^{10 / 3} \omega y_{0}^{2} y_{1}^{3}+3 a_{3}^{2} \omega y_{1}^{3}+a_{3}^{8 / 3} y_{0} y_{1}^{3}+ \\
& 2 a_{3}^{8 / 3} \omega y_{0} y_{1}^{3}+3 a_{3}^{4} \omega y_{0}^{2} y_{2}^{2} y_{1}^{2}+6 a_{3}^{10 / 3} \omega y_{0} y_{2}^{2} y_{1}^{2}-2 a_{3}^{10 / 3} y_{2}^{4} y_{1}- \\
& a_{3}^{10 / 3} \omega y_{2}^{4} y_{1}-3 a_{3}^{4} y_{0} y_{2}^{4} y_{1}-3 a_{3}^{8 / 3} y_{0}^{2} y 2 y 1-3 a_{3}^{8 / 3} \omega y_{0}^{2} y_{2} y_{1}- \\
& 6 a_{3}^{2} y_{0} y_{2} y_{1}-6 a_{3}^{2} \omega y_{0} y_{2} y_{1}-a_{3}^{4} y_{2}^{6}-a_{3}^{4} \omega y_{2}^{6}-a_{3}^{10 / 3} y_{0}^{2} y_{2}^{3}- \\
& 2 a_{3}^{10 / 3} \omega y_{0}^{2} y_{2}^{3}-3 a_{3}^{2} \omega y_{2}^{3}-a_{3}^{8 / 3} y_{0} y_{2}^{3}-2 a_{3}^{8 / 3} \omega y_{0} y_{2}^{3}+ \\
& \left.\left.3 a_{3}^{4 / 3} y_{0}^{2}+3 a_{3}^{2 / 3} y_{0}+3\right)\right)
\end{aligned}
$$


where $y_{0}+y_{1} \sqrt[3]{\omega^{-1}}+y_{2} \sqrt[3]{\omega^{-2}} \in K\left(\sqrt[3]{\omega^{-1}}\right)$ is such that $N_{K\left(\sqrt[3]{\omega^{-1}}\right) / K}(y)=a_{3}^{-2}$,

$$
\begin{aligned}
& \gamma_{2}=\frac{1}{a_{2}^{4}}\left(a_{2}^{4} a_{3}^{2} y_{1}^{6}-a_{2}^{10 / 3} a_{3}^{2} y_{2} y_{1}^{4}+a_{2}^{10 / 3} a_{3}^{2} \omega y_{2} y_{1}^{4}-3 a_{2}^{4} a_{3}^{2} y_{0} y_{2} y_{1}^{4}-\right. \\
& 3 a_{2}^{2} a_{3}^{3} y_{1}^{3}+a_{2}^{4} a_{3} y_{2}^{3} y_{1}^{3}-2 a_{2}^{10 / 3} a_{3}^{3} y_{0}^{2} y_{1}^{3}-a_{2}^{10 / 3} a_{3}^{3} \omega y_{0}^{2} y_{1}^{3}- \\
& 2 a_{2}^{8 / 3} a_{3}^{3} y_{0} y_{1}^{3}-a_{2}^{8 / 3} a_{3}^{3} \omega y_{0} y_{1}^{3}+3 a_{2}^{4} a_{3}^{2} y_{0}^{2} y_{2}^{2} y_{1}^{2}+ \\
& 6 a_{2}^{10 / 3} a_{3}^{2} y_{0} y_{2}^{2} y_{1}^{2}-2 a_{2}^{10 / 3} a_{3} y_{2}^{4} y_{1}-a_{2}^{10 / 3} a_{3} \omega y_{2}^{4} y_{1}- \\
& 3 a_{2}^{4} a_{3} y_{0} y_{2}^{4} y_{1}+3 a_{2}^{8 / 3} a_{3}^{3} y_{0}^{2} y_{2} y_{1}+6 a_{2}^{2} a_{3}^{3} y_{0} y_{2} y_{1}+a_{2}^{4} y_{2}^{6}+ \\
& 3 a_{3}^{4}-3 a_{2}^{2} a_{3}^{2} y_{2}^{3}-a_{2}^{10 / 3} a_{3}^{2} y_{0}^{2} y_{2}^{3}+a_{2}^{10 / 3} a_{3}^{2} \omega y_{0}^{2} y_{2}^{3}- \\
& \left.a_{2}^{8 / 3} a_{3}^{2} y_{0} y_{2}^{3}+a_{2}^{8 / 3} a_{3}^{2} \omega y_{0} y_{2}^{3}+3 a_{2}^{4 / 3} a_{3}^{4} y_{0}^{2}+3 a_{2}^{2 / 3} a_{3}^{4} y_{0}\right)
\end{aligned}
$$

where $y_{0}+y_{1} \sqrt[3]{a_{3}^{-1}}+y_{2} \sqrt[3]{a_{3}^{-2}} \in K\left(\sqrt[3]{a_{3}^{-1}}\right)$ is such that $N_{K\left(\sqrt[3]{a_{3}^{-1}}\right) / K}(y)=a_{2}^{-2}$ and

$$
\begin{aligned}
\gamma_{3}= & a_{1}^{4} a_{2}^{2} a_{3}^{4} y_{1}^{6}+a_{1}^{3} a_{2} a_{3}^{3} \sqrt[3]{a_{1} a_{2} a_{3}}(\omega-1) y_{2} y_{1}^{4}+ \\
& a_{1}^{2} a_{2} a_{3}^{2}\left(a_{1}^{2} a_{3}^{2} y_{2}^{3}-3\right) y_{1}^{3}-a_{1}^{3} a_{3}^{3} \sqrt[3]{a_{1} a_{2} a_{3}}(\omega+2) y_{2}^{4} y_{1}+a_{1}^{4} a_{3}^{4} y_{2}^{6}- \\
& 3 a_{1}^{2} a_{3}^{2} y_{2}^{3}+a_{1} a_{2} a_{3} \sqrt[3]{a_{1} a_{2} a_{3}} y_{0}^{2}\left(-a_{1}^{2} a_{2} a_{3}^{2}(\omega+2) y_{1}^{3}+\right. \\
& \left.3 a_{1}^{2} a_{3}^{2} \sqrt[3]{a_{1}^{2} a_{2}^{2} a_{3}^{2}} y_{2}^{2} y_{1}^{2}+a_{1} a_{3} \sqrt[3]{a_{1} a_{2} a_{3}} y_{2} y_{1}+a_{1}^{2} a_{3}^{2}(\omega-1) y_{2}^{3}+3\right)- \\
& \sqrt[3]{a_{1}^{2} a_{2}^{2} a_{3}^{2}} y_{0}\left(3 a_{1}^{3} a_{3}^{3} \sqrt[3]{a_{1} a_{2} a_{3}} y_{2} y_{1}^{4}+a_{1}^{2} a_{2} a_{3}^{2}(\omega+2) y_{1}^{3}-\right. \\
& 6 a_{1}^{2} a_{3}^{2} \sqrt[3]{a_{1}^{2} a_{2}^{2} a_{3}^{2}} y_{2}^{2} y_{1}^{2}+3 a_{1} a_{3} \sqrt[3]{a_{1} a_{2} a_{3}} y_{2}\left(a_{1}^{2} a_{3}^{2} y_{2}^{3}-2\right) y_{1}- \\
& \left.a_{1}^{2} a_{3}^{2}(\omega-1) y_{2}^{3}-3\right)+3
\end{aligned}
$$

where $y_{0}+y_{1} \sqrt[3]{a_{2}^{-1}}+y_{2} \sqrt[3]{a_{2}^{-2}} \in K\left(\sqrt[3]{a_{2}^{-1}}\right)$ is such that

$$
N_{K\left(\sqrt[3]{a_{2}^{-1}}\right) / K}(y)=\left(a_{1} a_{2} a_{3}\right)^{-1} .
$$

For $a_{1}=2, a_{2}=5$ and $a_{3}=3$ the values are:

$$
\begin{aligned}
& \gamma_{1}=9+6 \sqrt[3]{3}+4 \sqrt[3]{9} \\
& \gamma_{2}=8059+(1340 \omega+2040) \sqrt[3]{5}+(1876 \omega+2856) \sqrt[3]{25} \\
& \gamma_{3}=379+(116-32 \omega) \sqrt[3]{30}+(29-8 \omega) \sqrt[3]{900}
\end{aligned}
$$

\section{References}

[1] Crespo, T.: Explicit solutions to embedding problems associated to orthogonal Galois representations. J. Reine Angew. Math. 409 (1990), 180-189.

[2] Crespo, T.: Embedding Galois problems and reduced norms. Proc. Amer. Math. Soc. 112 (1991), 637-639.

[3] FröHlich, A.: Orthogonal representations of Galois groups, StiefelWhitney classes and Hasse-Witt invariants. J. Reine Angew. Math. 360 (1985), 84-123. 
[4] Grundman, H. G., Smith, T. L. and Swallow, J. R.: Groups of order 16 as Galois Groups. Expositio Math. 13 (1995), 289-319.

[5] Massy, R.: Solutions explicites de problèmes de plongement. J. Number Theory 20 (1985), 299-314.

[6] Serre, J. P.: Corps locaux, 10ème ed. Actualités Scientifiques et Industrielles 1296. Hermann, Paris, 1968.

[7] Vela, M.: Explicit solutions of Galois embedding problems by means of generalized Clifford algebras. J. Symbolic Comput. 30 (2000), 811-842.

Recibido: 18 de noviembre de 2002

Revisado: 29 de mayo de 2003

Montserrat Vela

Departament de Matemàtica Aplicada II

Universitat Politècnica de Catalunya

C/ Jordi Girona, 1-3

Edifici Omega, Campus Nord

E-08034 Barcelona, Spain

Montse.Vela@upc.edu

Partially supported by MECD grant BFM2000-0794-C02-01 and AGAUR 2002SGR 00148. 